\title{
Understanding and Tuning the Intrinsic Hydrophobicity of Rare- Earth Oxides: A DFT+U Study
}

\author{
Giuliano Carchini, ${ }^{\dagger}$ Max García-Melchor, ${ }^{\ddagger}{\text { Zbigniew } \text { Lodziana, }^{\S} \text { and Núria López }}^{*}, \|$ \\ ${ }^{\dagger}$ ICIQ - Institute of Chemical Research of Catalonia, The Barcelona Institute of Science and Technology, Av. Països Catalans 16, \\ 43007 Tarragona, Spain \\ ${ }^{\ddagger}$ SUNCAT Center for Interface Science and Catalysis, SLAC National Accelerator Laboratory, Menlo Park, California 94025, United \\ States \\ ${ }^{\S}$ Institute of Nuclear Physics, Polish Academy of Sciences, ulica Radzikowskiego 152, PL-31-342 Krakow, Poland \\ "ICIQ - Institute of Chemical Research of Catalonia, Av. Països Catalans 16, 43007 Tarragona, Spain
}

\begin{abstract}
Rare-earth oxides (REOs) possess a remarkable intrinsic hydrophobicity, making them candidates for a myriad of applications. Although the superhydrophobicity of REOs has been explored experimentally, the atomistic details of the structure at the oxide-water interface are still not well understood. In this work, we report a density functional theory study of the interaction between water and $\mathrm{CeO}_{2}, \mathrm{Nd}_{2} \mathrm{O}_{3}$, and $\alpha-\mathrm{Al}_{2} \mathrm{O}_{3}$ to explain their different wettability. The wetting of the metal oxide surface is controlled by geometric and electronic factors. While the electronic term is related to the acid-base properties of the surface layer, the geometric factor depends on the matching between adsorption sites and oxygen atoms from the hexagonal water network. For all the metal oxides considered here, water dissociation is confined to the first

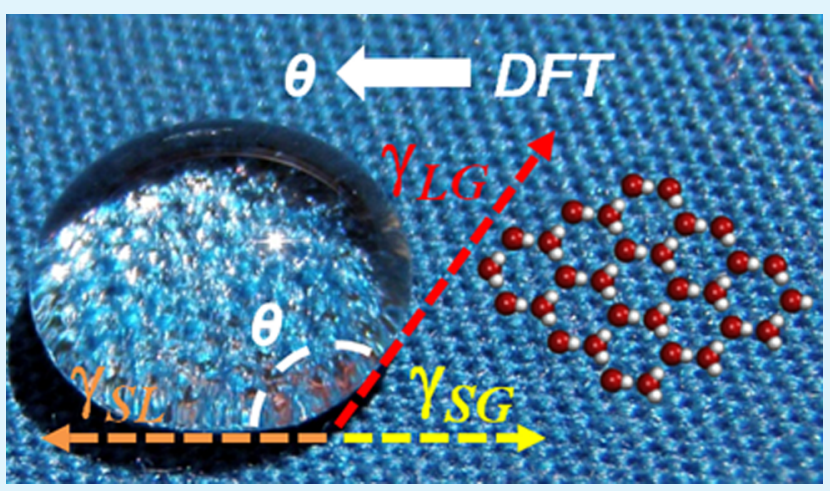
oxide-water layer. Hydroxyl groups on $\alpha-\mathrm{Al}_{2} \mathrm{O}_{3}$ are responsible for the strong oxide-water interaction, and thus, both $\mathrm{Al}-$ and hydroxyl-terminated wet. On $\mathrm{CeO}_{2}$, the intrinsic hydrophobicity of the clean surface disappears when lattice hydroxyl groups (created by the reaction of water with oxygen vacancies) are present as they dominate the interaction and drive wetting. Therefore, hydroxyls may convert a intrinsic nonwetting surface into a wetting one. Finally, we also report that surface modifications, like cation substitution, do not change the acid-base character of the surface, and thus they show the same nonwetting properties as native $\mathrm{CeO}_{2}$ or $\mathrm{Nd}_{2} \mathrm{O}_{3}$.
\end{abstract}

KEYWORDS: oxide, water interface, density functional theory, wetting, defects

\section{INTRODUCTION}

The precise control of the wettability of a material is crucial for its application into a wide range of technological problems. ${ }^{1-5}$ Therefore, materials have been classified in terms of this parameter. Superhydrophobic surfaces (full nonwetting) are characterized by contact angles $(\theta$, between water and the surface) larger than $150^{\circ}$, whereas superhydrophilic ones show contact angles close to zero degrees, with a full range of intermediate behaviors. Hydrophobic coatings are fundamental to many mechanical parts in industry that need to be thermally and mechanically resistant, ${ }^{6-8}$ while hydrophilicity is required for self-cleaning purposes. ${ }^{9,10}$ Recently, the degree of wettability has been found to correlate with the viability of electrochemical processes required in the oxygen evolution reaction (OER), crucial for the successful development of new energy sources. ${ }^{11}$

Complex textures and architectures have been employed to reduce the ability of water to wet surfaces, while much less attention has been focused on the intrinsic mechanisms that control wetting on oxides at the atomistic level. However, both the surface geometry and the chemical composition are known to be crucial for the wetting behavior. Recently, Azimi et al. ${ }^{12}$ found experimentally that a strong hydrophobicity is intrinsic to the rare-earth oxides (REOs), ${ }^{13}$ and multiple applications have been suggested. ${ }^{14,15}$ Thus, REOs behave differently than other oxides that also exhibit hexagonal surface terminations, such as the $\alpha$-alumina which wets completely.

An explanation for the strong hydrophobicity of REOs has been suggested, pointing out to the fact that the affinity of the different materials for water is primarily based on their electronic structure. ${ }^{12}$ According to this interpretation, a hydrophilic material presents empty metal orbitals available to accept electrons from the water oxygens, whereas a hydrophobic material is characterized by nonaccessible metal orbitals, which result in a minor water affinity, as shown in Figure 1. 

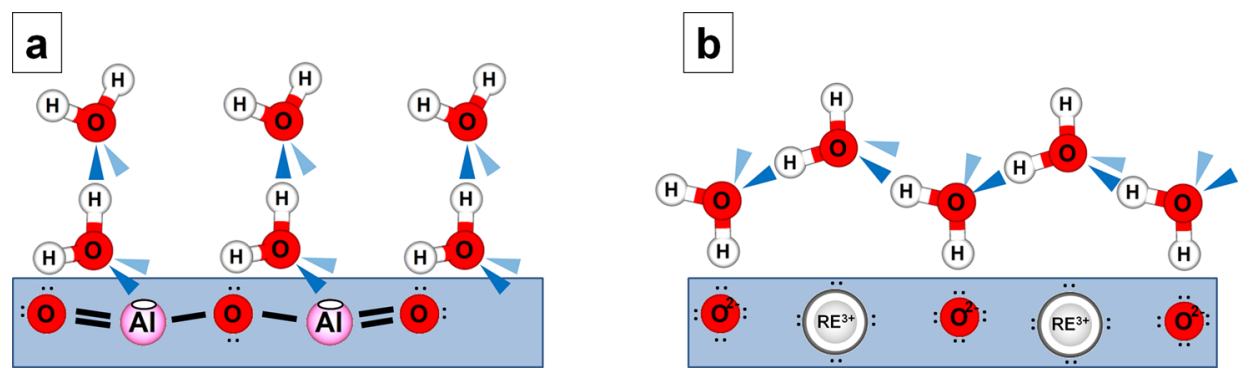

Figure 1. Proposed model of the interaction of water at the interface with two prototypical oxides: (a) $\alpha$-alumina and (b) REOs. Adapted from ref 12.

This interpretation has been also extrapolated to $\mathrm{TiO}_{2}$ anatase thin films. ${ }^{16}$ However, in all these cases, the proposed structures of the interfaces and the water layers ${ }^{12}$ do not agree with the atomistic description of the adsorption of single water molecules ${ }^{17}$ or different water layer coverages, ${ }^{18}$ for which partial dissociation has been found. The energies reported in these works agree with the recent calorimetric values reported for adsorption in the group of Navrotsky. ${ }^{19}$ However, the interpretation of water adsorption on ceria through theoretical means is still under debate. Some authors have pointed out that if the reservoir is gas-phase water and entropy is considered, then water adsorption would be only favored at $10^{5} \mathrm{~atm}^{20}$ which is clearly against experiments. ${ }^{19}$ Moreover, general descriptions of the water-material interface shows a detailed balance between electrostatic forces, H-bonds, and hydroxyl groups on the surface. ${ }^{21}$

The reduction of the surface has been also proposed to be a key parameter modifying the wettability of REOs. ${ }^{5,22}$ In particular, for $\mathrm{CeO}_{2}$, it was found that once oxygen vacancies are present, full wetting appears. This behavior is common to other oxides like the nonwetting anatase $\left(\theta=110^{\circ}\right)$, which becomes completely hydrophilic upon irradiation; the process is reversible if the surface is kept under ambient conditions for one week ${ }^{16}$ and also reported for $\mathrm{ZnO}$ nanomaterials. ${ }^{23}$

In the present work, density functional theory (DFT) simulations have been employed to study the intrinsic properties of wetting on oxides: two REOs and $\alpha-\mathrm{Al}_{2} \mathrm{O}_{3}$. This kind of simulations have previously been able to unravel the origin of properties related to the interaction of water with metals, for which a large amount of experimental data has been collected; ${ }^{24}$ this knowledge has been transferred to more reactive surfaces like oxides. ${ }^{18,25-30}$ The polarity of the surface and reorientation of the interfacial water are usually taken as driving forces for the hydrophobic/hydrophilic surface character. ${ }^{6,21,31,32}$ For instance, hydrophobic properties have been for long related to weak hydrogen bonding to the surface and strong orientation effects. ${ }^{33}$ The experimental contact angle $\theta$ parameter can be obtained through the Young equation, where the only required input is the adsorption energy of water at the interface. This approach was successfully used to analyze the wetting of nanoparticles on reactive oxides. ${ }^{34-38}$

The aim of our work is to unravel the chemical origin of the wetting properties of a set of REOs and compare it to that simple oxides. Herein, we find the main descriptors for wettability and demonstrate that the hydrophobic-hydrophilic character of the material can be tuned by modifying its surface structure. Our results point out to two main factors responsible for the wetting properties, namely geometric and electronic contributions. Finally, we have also addressed the way how these governing contributions can be tuned to obtain a desired wettability.

\section{METHODOLOGY}

All the DFT calculations reported in this work were carried out using VASP code, version 5.3.3. ${ }^{39,40}$ The $\mathrm{PBE}^{41}$ functional was used to obtain the energies. In the setup, PAW pseudopotentials ${ }^{42}$ were employed to represent the core electrons, whereas the valence electrons were expanded in plane waves with a cutoff energy of $500 \mathrm{eV}$. For the RE metals, the f-electrons belong to the valence (thus Ce the valence contains $4 \mathrm{f}, 5 \mathrm{~d}, 6 \mathrm{~s}$ and for $\mathrm{Nd} 4 \mathrm{f}$ and $6 \mathrm{~s})$. Spin polarization was introduced when needed. Slab calculations were performed with a vacuum space of at least $12 \AA$ along the $z$ direction. In all the calculations, the electronic structure was converged up to a $10^{-5} \mathrm{eV}$ threshold, while geometries were optimized up to an energy criteria of $10^{-3} \mathrm{eV}$. The hexagonal lattice of ice water was used as initial structure to investigate adsorption of one or two water bilayers. ${ }^{43}$ The corresponding surface energy was calculated for a slab of 5 bilayers $(7 \times 7 \times 1$ k-point $)$; and obtaining a value of $0.044 \mathrm{eV} / \AA^{2}$.

$\alpha-\mathrm{Al}_{2} \mathrm{O}_{3}$ is characterized by a rhombohedral unit cell with a $R \overline{3} c$ symmetry (corundum). The computed lattice constants of $a_{\text {calc }}=4.806$ $\AA$ and $c_{\text {calc }}=13.114 \AA$ (using a $5 \times 5 \times 2$ k-point) are in good agreement with the experimental values of $a_{\text {exp }}=4.755 \AA$ and $c_{\exp }=$ $12.991 \AA^{44}$ To simulate this material, we considered the most stable facet (0001) using a $2 \times 2$ supercell consisting of a slab of 18 layers and the sampling of the Brillouin zone with a $5 \times 5 \times 1$ k-point grid. The bottom two layers were fixed to their bulk positions while the rest were allowed to relax. In vacuum, the Al-terminated surface relaxes extensively with the cations moving toward the underlying anions; this termination has the lowest surface energy. ${ }^{45}$ However, this surface is very reactive, and the terminal $\mathrm{Al}$ atoms dissociate water molecules forming solvated $\mathrm{Al}(\mathrm{OH})_{3}$, which can be easily removed from the surface, leading to an Al-deficient surface. ${ }^{46}$ As a result, the equilibrium surface termination in the presence of water corresponds to a fully hydroxylated layer, $\mathrm{O}_{\mathrm{lat}} \mathrm{H}^{47,48}$ In this work, the adsorption of one and two water bilayers on both surface terminations was investigated.

Cerium dioxide has a cubic structure with a $F m \overline{3} m$ symmetry, cubic fluorite. The optimized lattice parameter using a $7 \times 7 \times 7$ k-point mesh was $a_{\text {calc }}=5.497 \AA,{ }^{49}$ which is in good agreement with the experimental value of $a_{\exp }=5.411 \AA .^{50}$ In the REOs, there are localized f-electrons; therefore, a GGA+U scheme was used, since standard DFT functionals cannot describe them correctly; $U_{\text {eff }}=4.5 \mathrm{eV}$ (Ce in $\mathrm{CeO}_{2}$ ) has been proven to be a reasonable choice. ${ }^{51,52}$ As for the surface, we modeled the most stable (111) facet with a $(2 \times 2)$ periodicity which consists of three $\mathrm{O}-\mathrm{Ce}-\mathrm{O}$ trilayers. On the hexagonal surface represented by the $\mathrm{CeO}_{2}(111)$, an ice bilayer can be adsorbed. Similarly to fcc metals, ${ }^{24,43}$ the water bilayer almost matches the position of the acid centers, and thus dissociation as for reactive metals can be expected. In the present case, both acid and basic centers would end up as hydroxyl groups. On the $\mathrm{p}(2 \times 2)$ supercell, a total of eight water molecules represents the bilayer. Among all possible orientations of the water bilayer, only those with a $\mathrm{H}$-up configuration have been taken into account. The reason for this is that all the basic centers on the surface are compromised by the potential dissociation of the very first four water molecules that are directly in contact with 
the surface. This intrinsic water bilayer can adsorb consecutive water layers if more water is available. The model with two bilayers has been taken as representative of the interaction at the surface, since for further layer adsorption the water liquid nature would be more relevant to accommodate dangling $\mathrm{H}$-bond structures. We have assessed that the energy variation on the ice structure by introducing lateral expansion/contractions is below $70 \mathrm{meV}$, thus in agreement with the pseudomorphic growth proposed here. This assumption follows the Brunauer-Emmett-Teller theory of multilayer adsorption of a differential between first layers and further multilayer adsorption. ${ }^{53} \mathrm{CeO}_{2}$ (111) surface studies were carried out with $3 \times$ $3 \times 1$ k-points and the bottommost $\mathrm{O}-\mathrm{Ce}-\mathrm{O}$ trilayer was fixed to mimic the bulk, while the topmost two $\mathrm{O}-\mathrm{Ce}-\mathrm{O}$ trilayers were allowed to relax. The approach used in this work to calculate water adsorption energies turns out to be sufficiently accurate and they are in the agreement with the experiments in ref 19. The van der Waals contributions were indicated to be at most $180 \mathrm{meV}$ for a single water molecule. ${ }^{17}$ This contribution is similar to the increase in cohesive energy of lattice ice. ${ }^{43}$

The state of the surface (stoichiometry and geometry) drives the wetting properties. ${ }^{22} \mathrm{CeO}_{2}$ can lose oxygen quite easily (forming vacancies); this property is closely related to the oxygen storage capacity of the material. Vacancy formation is energetically more favorable in the bulk than on the surface, ${ }^{52}$ but these vacancies are dynamic. ${ }^{54}$ When they are on the surface, they can be healed by oxygen gas in the environment. Extraction and healing of vacancies by reactive gases is also common to other oxides. ${ }^{55}$ In our case, the model to represent this situation has been set in the following way. In the $\mathrm{p}(2$ $\times 2$ ) supercell, one of the surface oxygen atoms has been removed from the surface, then this empty site has been filled with a hydroxyl group. Therefore, one of the Ce atoms nearby has been effectively reduced to $\mathrm{Ce}^{3+}$ and the lattice hydroxyl concentration is $0.25 \mathrm{ML}$. Moreover, we have assessed the changes induced by doping $\mathrm{CeO}_{2}$. The setup of these calculations is as follows: a dopant cation replaced $\mathrm{Ce}$ in the bulk of the material, and the bulk was reoptimized for two typical dopants, $\mathrm{La}$ and $\mathrm{Zr}$, at a concentration of $8 \%$. The (111) surfaces were then reoptimized. Given the segregation patterns of the impurities on this surface, two different configurations were considered for La and Zr. La stays preferentially on the surface due to its large ionic radius, while for $\mathrm{Zr}$ both situations (bulk or surface substitution) are almost equivalent energetically and thus entropic contributions would favor insertion of $\mathrm{Zr}$ in the bulk of the material. ${ }^{56}$

A second REO neodymium oxide, was also considered in this study. It is characterized by a hexagonal unit cell with a $P \overline{3} m 1$ symmetry. In this case, the $U_{\text {eff }}$ parameter was set to $5.44 \mathrm{eV} .^{51}$ The optimized cell parameters, $a_{\text {calc }}=3.859 \AA$ and $c_{\text {calc }}=6.124 \AA$, are in agreement with the experimental values of $a_{\exp }=3.829 \AA$ and $c_{\exp }=5.997 \AA .{ }^{57}$ The most stable (0001) facet was taken to illustrate adsorption with a $(2 \times$ 2 ) supercell and a $3 \times 3 \times 1 \mathrm{k}$-point mesh. During the optimization of the different structures, we fixed the bottom layer of $\mathrm{Nd}$ and $\mathrm{O}$ as part of the bulk, while the rest was allowed to relax. The terminal oxygens in the bottom layer were capped with $\mathrm{H}$ atoms in order to remove the spurious contributions to the electronic structure due to the polar nature of the surface. Water adsorption on this surface was explored in the same way as on $\mathrm{CeO}_{2}(111)$.

The adsorption energy of ice water bilayers to the surfaces was obtained as follows:

$$
E_{\text {ads }}^{\text {ice }}(\text { Nlayers })=E_{\text {tot }}-E_{\text {ice }}(\text { Nlayers })-E_{\text {surf }}
$$

where $E_{\text {tot }}, E_{\text {ice }}$ (Nlayers), and $E_{\text {surf }}$ are, respectively, the energies of the total system, an $N$-layer standing ice structure $(N$ can be either 2 or 4 , thus representing a bilayer and a double bilayer) and the bare oxide surface, exothermic processes are indicated by negative values.

The affinity of a material for water is expressed as the contact angle between the liquid and the surface of the material; see Figure 2. The contact angle can be related to the surface energies of different interfacial pairs through the Young equation: ${ }^{58}$
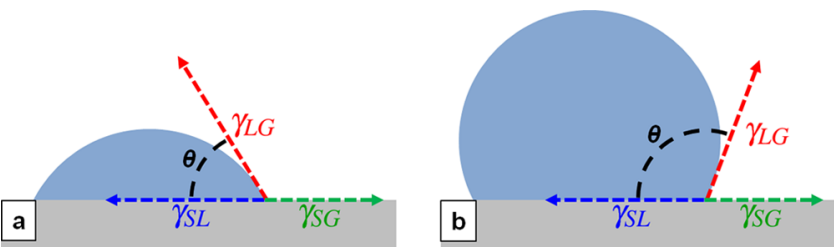

Figure 2. Sketch of the interaction between a liquid and a surface. The contact angle $\theta$ is small for hydrophilic materials (a) and large for hydrophobic ones (b). $\gamma_{X Y}$ correspond to the surface energies of the different pairs: SL, solid-liquid; SG, solid-gas; and LG, liquid-gas.

$$
\cos (\theta)=\frac{\gamma_{\mathrm{SG}}-\gamma_{\mathrm{SL}}}{\gamma_{\mathrm{LG}}}
$$

where $\gamma_{X Y}$ represents the surface energy at a given interface, and $\mathrm{S}, \mathrm{L}$, and $\mathrm{G}$ stand for solid, liquid, and gas phase, respectively. It has to be noted that only one thermodynamically stable contact angle is possible for an ideal solid surface. For such simple cases, there is zero contact angle hysteresis; that is, the advancing and receding contact angles are equal. ${ }^{59}$ This is precisely what the DFT-based Young calculations can provide. Experimental results have identified that there is a strong correlation between the average strength of ice adhesion and the receding contact angle of water. ${ }^{1}$ Indeed, the surface energy of water and ice are comparable. ${ }^{60}$ Based on this, and the fact that theoretical simulations represent icelike structures better than liquid ones, ice models have been used as surrogate for liquid water. Therefore, the surface energy $\gamma_{\text {ice }}$ is taken throughout the present article.

Equation 2 can be rewritten in terms of the adsorption energy of ice as

$$
\gamma_{\mathrm{SL}}=\gamma_{\mathrm{SG}}+\frac{E_{\mathrm{ads}}^{\mathrm{ice}}(\text { Nlayer })}{A}=\gamma_{\mathrm{SG}}+\gamma_{\text {int }}(\text { Nlayer })
$$

where $\gamma_{\text {int }}$ (Nlayer) is the surface energy corresponding to the interaction between the ice of $N$-layers and the surface. From this equation, the contact angle can be obtained as

$$
\cos (\theta)=\frac{-E_{\text {ads }}^{\mathrm{ice}}(\text { Nlayer })}{A \gamma_{\mathrm{LG}}}=\frac{-E_{\mathrm{ads}}^{\mathrm{ice}}(\text { Nlayer })}{A \gamma_{\text {ice }}}
$$

Thus, from the density functional theory calculations, $\gamma_{\text {ice }}$, the adsorption energy, $E_{\text {ads }}^{\text {ice }}$, and the surface area, $A$, can be employed to obtain the contact angle, which is directly comparable to experiments. A similar approach was employed by Lange and co-workers to illustrate the wetting on $\mathrm{Si}(111) .^{61}$

\section{RESULTS}

The two relevant energy parameters are related to the water dissociation degree at the interface (Table 1) and the interfacial (adsorption) energy contribution (Table 2). Water adsorption

Table 1. Adsorption Energies Per Water Molecule of the Water Layers, $E_{\text {ads }}$, in $\mathrm{eV} / \mathrm{H}_{2} \mathrm{O}$ with Respect to Isolated Water Molecules, as a Function of the Degree of Dissociation for the First Water Bilayer $(2 \mathrm{~L}=8 \text { Molecules })^{a}$

$\begin{array}{cccc}\begin{array}{c}\text { fraction of dissociated water } \\ \text { molecules }\end{array} & \alpha-\mathrm{Al}_{2} \mathrm{O}_{3}(0001) & \mathrm{CeO}_{2}(111) & \mathrm{Nd}_{2} \mathrm{O}_{3}(0001) \\ 0 / 8 & -0.98 & -0.20 & -0.31 \\ 1 / 8 & -1.11 & -0.39 & -0.27 \\ 2 / 8 & -\mathbf{1 . 2 1} & -\mathbf{0 . 4 2} & -\mathbf{0 . 5 5} \\ 3 / 8 & -0.86 & -0.37 & 2 / 8 \\ 4 / 8 & -1.16 & -0.35 & 2 / 8\end{array}$

${ }^{a}$ Values in bold indicate the most stable structures. For $\mathrm{Nd}_{2} \mathrm{O}_{3}$, the structures with more than two dissociated waters in the initial configuration revert to $2 / 8$ configurations (marked as $2 / 8$ ). 
Table 2. Water Adsorption Energies, $\gamma_{\text {int }} \mathrm{eV} / \AA^{2}$, with Respect to the Isolated Bilayer or Double Bilayer, on the Different Oxides

\begin{tabular}{cccr} 
oxide & termination & $2 \mathrm{~L}$ & \multicolumn{1}{c}{$4 \mathrm{~L}$} \\
$\mathrm{Al}_{2} \mathrm{O}_{3}$ & $\mathrm{Al}$ & -0.062 & -0.065 \\
$\mathrm{Al}_{2} \mathrm{O}_{3}$ & $\mathrm{O}_{\mathrm{lat}} \mathrm{H}$ & -0.058 & -0.055 \\
$\mathrm{CeO}_{2}$ & & -0.011 & 0.006 \\
$\mathrm{CeO}_{2}$ & $\mathrm{O}_{\text {lat }} \mathrm{H}$ & -0.043 & -0.053 \\
$\mathrm{Nd}_{2} \mathrm{O}_{3}$ & & -0.007 & 0.009 \\
\hline
\end{tabular}

on the Al-terminated $\alpha-\mathrm{Al}_{2} \mathrm{O}_{3}$ surface is exothermic and close to $1 \mathrm{eV} / \mathrm{H}_{2} \mathrm{O}$ for the first layer; see Table 1 and Figure 3. The equilibrium state for the first water bilayer leads to about $50 \%$ dissociation of the water molecules directly in contact with the surface. This structure presents water molecules with a H-down orientation that dissociate on the surface. They are forming $\mathrm{OH}$ groups with the lattice atoms which interact to other $\mathrm{OH}$ groups from the incoming water, in agreement with previous theoretical works. ${ }^{46}$ The corrugation of the first water layer, that is, the distance between the planes defined by the horizontal and the vertical water molecules, is $0.765 \AA$. Further adsorption on top of this first layer is also possible with a large interaction energy, leading to a configuration where $\mathrm{H}$-bonds between the $\mathrm{H}$-down water molecules on the surface are present. The resulting water structure preserves most of the characteristics of ice, and even the geometric distortion induced by the acid-base patterning of the surface is small. As a consequence, the corrugation between $\mathrm{O}$ atoms belonging to the second layer is $0.548 \AA$.

However, the reaction of water with the alumina surface is known to alter the surface itself by eliminating $\mathrm{Al}$ cations to the solution due to the formation of soluble species. The ultimate consequence is that, in the presence of water, the surface contains less $\mathrm{Al}$ and the oxygens coordinating the cation end up as hydroxyl groups. Thus, the resting state is an $\mathrm{O}_{\mathrm{lat}} \mathrm{H}$ terminated surface (Figure 3d). ${ }^{46}$ Further water adsorption on $\mathrm{O}_{\text {lat }} \mathrm{H}$ occurs in a slightly different manner than for the $\mathrm{Al}$ terminated surface. In this case, the most stable bilayer adsorbs bending some of the $\mathrm{O}_{\text {lat }} \mathrm{H}$ (see Figure 3e,f), which are not pointing toward the vacuum but to other $\mathrm{O}$ atoms in the lattice. The incoming water bilayer interacts with the basic oxygen lone pair of the flat water molecules directly connected to the $\mathrm{O}_{\text {lat }} \mathrm{H}$ pointing to the vacuum, while the $\mathrm{O}_{\mathrm{lat}} \mathrm{H}$ bend to accommodate the perpendicular $\mathrm{H}$-down water molecules from the bilayer better. The energy penalty is counterbalanced by the new $\mathrm{H}$ bonds established between all the $\mathrm{O}_{\mathrm{lat}} \mathrm{H}$ centers and the water molecules of the incoming bilayer. The bilayer strongly interacts with the material, the distances to the surface atoms are 2.329 and $3.094 \AA$; the corrugation of the water bilayer is $0.540 \AA$. Thus, the first bilayer in contact to the surface presents H-down waters. This type of configurations do not accommodate further water. ${ }^{21}$ Therefore, the interfacial layer is hydrophobic with respect to further water adsorption. Indeed, water adsorption of the second water bilayer is close to thermoneutral, $0.02 \mathrm{eV} / \mathrm{H}_{2} \mathrm{O}$ molecule, when calculated with respect to the isolated bilayer. In this final configuration the corrugation of the second bilayer is $0.539 \AA$. Therefore, the large interaction energies observed for the first water bilayer on $\alpha-\mathrm{Al}_{2} \mathrm{O}_{3}$ surface results in wetting angles of $0^{\circ}$, in reasonable agreement with the experimental determination of $10^{\circ} . .^{12}$

Similarly to $\alpha-\mathrm{Al}_{2} \mathrm{O}_{3}$, the adsorption of the first water bilayer on a clean $\mathrm{CeO}_{2}$ (111) surface can occur dissociatively; see Figure 4. In this case, however, our results indicate that only $50 \%$ of the molecules in direct contact with the surface dissociate (Table 1). This behavior is in line with previous investigations showing that water can partially dissociate on oxides. $^{62}$ The influence of the coverage on the dissociative/ associative equilibrium of the water adsorption has been also investigated. ${ }^{18}$ From our calculations, for the partially dissociated structure, the average adsorption energy for the first bilayer is significantly smaller than on alumina $(-0.42 \mathrm{eV} /$ $\mathrm{H}_{2} \mathrm{O}$ ). The adsorbed bilayer is slightly compressed so as to match the templating effect of the acid-base centers on the surface. For real surfaces, provided that compression is too large like water on $\mathrm{FeO},{ }^{30}$ large Moire patterns could appear. However, for the compression ranges explored in the present work we have considered that the registry between the ice layer

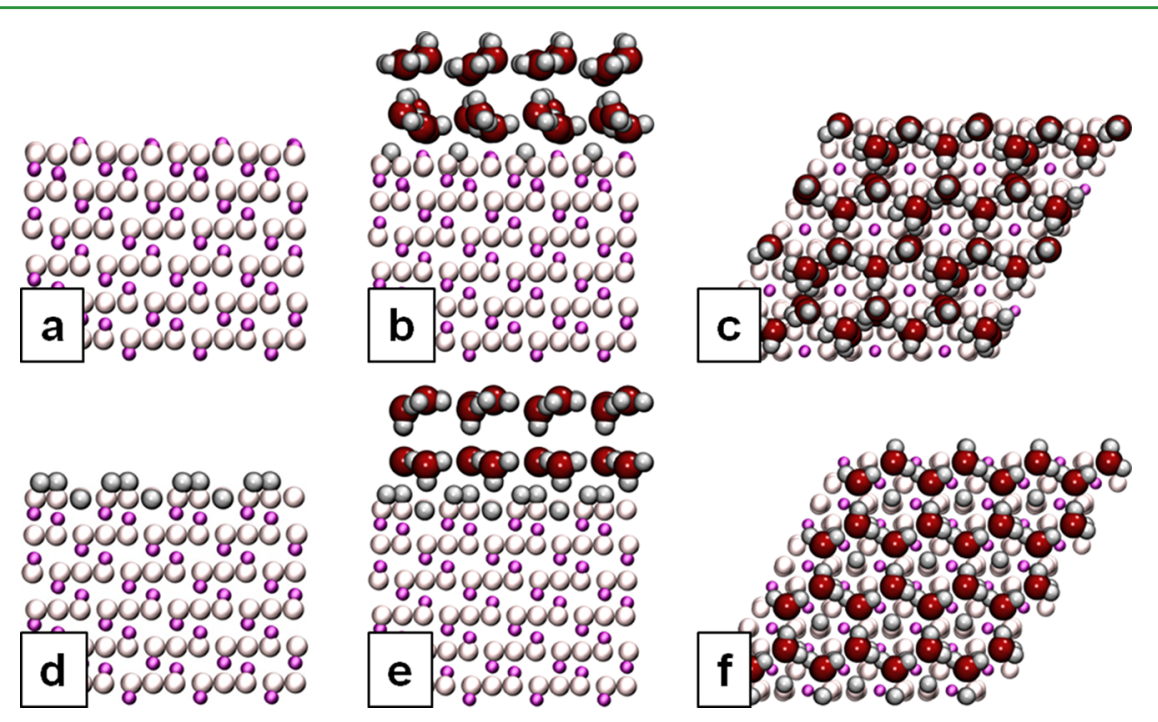

Figure 3. $\mathrm{Al}$ terminated $\alpha-\mathrm{Al}_{2} \mathrm{O}_{3}(0001)$ clean (a), side (b), and top (c) view. Panels $(\mathrm{b}-\mathrm{e})$ correspond to the side of the systems with two bilayers adsorbed on top, and $(\mathrm{e}, \mathrm{f})$ the corresponding top view. Some of the water molecules in contact with the surface are dissociated. $\mathrm{O}_{\mathrm{lat}} \mathrm{H}$-terminated $\alpha$ $\mathrm{Al}_{2} \mathrm{O}_{3}(\mathrm{~d})$, side (e), and top (f) view. The hydroxylated surface determines the flipping of the $\mathrm{H}$-up molecules to $\mathrm{H}$-down. The color codes are small spheres for the oxide (red, oxygens; rest, metal cations) and large spheres for $\mathrm{H}_{2} \mathrm{O}$ (red, oxygen; white, hydrogen). 

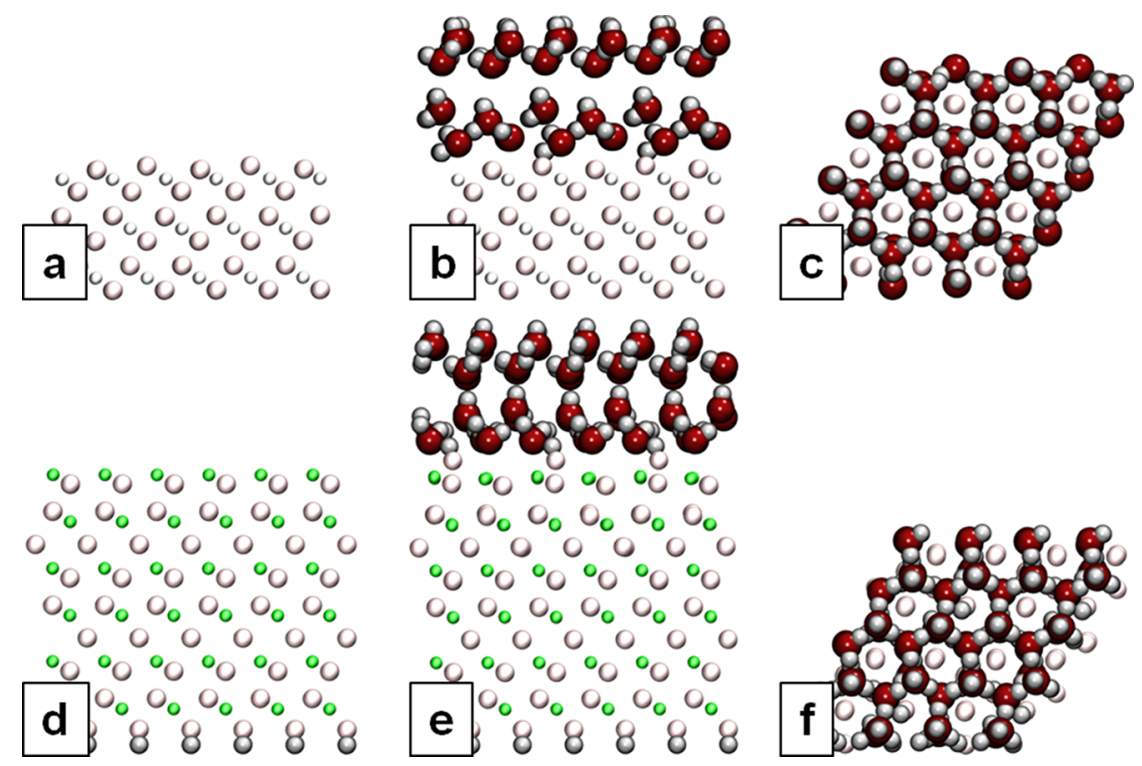

Figure 4. $\mathrm{CeO}_{2}(111)$ clean surface (a), side (b), and top (c) view in the presence of water (clean (d), side (e), and top (f) view of $\mathrm{Nd}_{2} \mathrm{O}_{3}(0001)$ ). To avoid spurious terms, the polar termination of the bottom layer of $\mathrm{Nd}_{2} \mathrm{O}_{3}$ was capped with $\mathrm{H}$ atoms. Due to the shorter distance between the metals in the lattice, the ice pattern turns out to be more compressed, and hence, the interaction with the surface is weaker compared to alumina.

and the surface would be well characterized with the present methodology. As the horizontally aligned water molecules are the ones dissociating on the surface, the perpendicular ones that close the bilayer are pointing with the $\mathrm{H}$-up toward the vacuum. Due to the dissociation, both H-down and H-up vertical molecules appear. The corrugation between the oxygen of the layer is large and equals $1.588 \AA$. The degree of dissociation can be understood by the competition between the acid-base characteristics of the surface and the geometric constraints induced by the arrangement of these centers on the surface. Lattice compressions disfavor dissociated states, since the oxygens on the surface are more basic, ${ }^{63}$ but this is counterbalanced by the missing resonances of the sixmembered water rings in ice, more exothermic under stress. ${ }^{43}$ The combination of both effects control the dissociation degree. Further adsorption of the second bilayer follows the ice patterns on the H-up from the first bilayer. The computed adsorption energy per water molecule is quite endothermic (ca. $0.2 \mathrm{eV}$ ) due to the large compression of the layer, as demonstrated by the $\mathrm{O}-\mathrm{O}$ distance in the outermost layer of $3.863 \AA$. This adsorption energy corresponds to a contact angle of $100^{\circ}$, in remarkable agreement with the reported experimental value of $103 \pm 3^{\circ} .^{12}$

For $\mathrm{Nd}_{2} \mathrm{O}_{3}$, we have found that this REO behaves similarly to $\mathrm{CeO}_{2}$. At the interfacial layer partial dissociation is observed, with an adsorption energy close to that of $\mathrm{CeO}_{2}$. For the adsorbed bilayer, water dissociation over $50 \%$ does not result in stable configurations. The corrugations in the case of the bilayer and double bilayers are 1.577 and $1.654 \AA$, respectively. The resulting contact angle is $103^{\circ}$, again in the range determined experimentally, $101^{\circ} .12$

Therefore, our results indicate that, at least for the native surfaces, the methodology presented here can reproduce the experimental contact angles.

\section{DISCUSSION}

Origin of Hydrophobicity. Varanasi et al. ${ }^{12}$ rationalized the hydrophilic/hydrophobic character of REOs with the accessibility of the metal empty orbitals to the oxygens from water (see Figure 1). The first-principles simulations presented here indicate a more complex interface, where water adsorption can range from dissociated to partially dissociated state. Thus, cation accessibility shall be accounted together with other geometric factors. Moreover, simulations suggest a negligible influence of the surface electronic effects on the adsorption of the second and subsequent layers.

On alumina, both inspected surfaces ( $\mathrm{Al}$ and $\mathrm{O}_{\mathrm{lat}} \mathrm{H}$ terminated) lead to full wetting. As for the Al-terminated surface, dissociation occurs and a strong water/surface interaction appears. The subsequent water layers adsorbed on top closely resemble the ice structure, as the $\alpha-\mathrm{Al}_{2} \mathrm{O}_{3}$ and ice lattices do not differ much (4.806 and $4.771 \AA$, respectively). In summary, the strong interaction at the interface drives the wettability. On the other hand, in the hydroxyl terminated alumina surface there is a large rearrangement of the surface hydroxyl groups, implying that further water adsorption prefers strong H-down configuration, establishing all possible hydrogen bonds. This determines a strong interaction and therefore full wetting of the surface.

For the REOs the situation is different: the lateral dimensions of the oxide lattice are smaller than those of ice, although still in the regime that allows a tetragonal distortion (i.e., trying to the register with the surface cations allows a more or less tridimensional distortion). The oxide lattice is therefore smaller with respect to that of the ice (4.806 $\AA$ ice vs $3.863 \AA$ for $\mathrm{CeO}_{2}$ ). When it acts as a template, it does not allow water to benefit fully from the acid-base character of the metal oxide surface; in fact, there is not enough space to accommodate a full set of dissociated water molecules. This leads to water-surface interfaces that are partially dissociated bilayers (and likely quite dynamic), with fragments appearing both on the surface lattice anions and cations. Besides, further water adsorption is screened from the surface properties. Hence, for the external water layers, only the geometric constraint templates from the oxide are responsible for the wetting characteristics, see Figure 4. For this reason, the contact angle is found to depend only on the cation-cation distance on the surface, see Figure 5. 


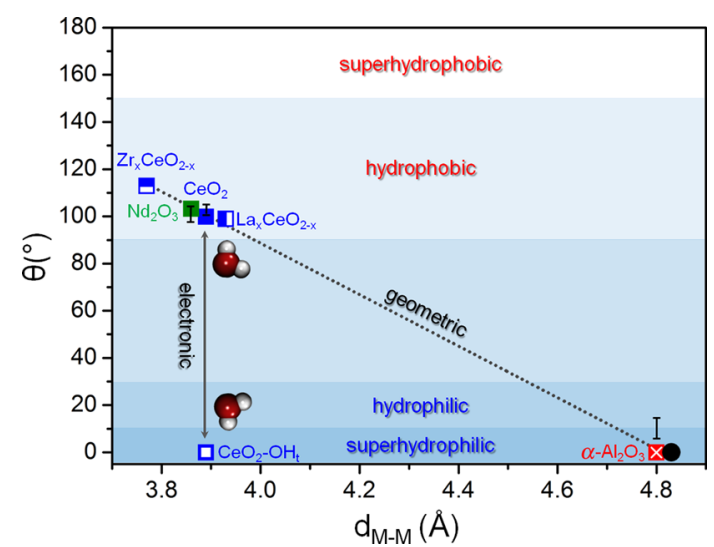

Figure 5. Contact angle, $\theta$ in degrees, as a function of the surface metal-metal distance, $d_{\mathrm{M}-\mathrm{M}}$ in $\AA$. Experimental values (marked as error bars) included in the graph have been taken from ref 12 . The dotted line illustrates the geometric contribution and corresponds to the linear fitting of all the clean surface points, but shall be taken as a guide to the eye. The arrow indicates the electronic effects. Notice that, for $\alpha-\mathrm{Al}_{2} \mathrm{O}_{3}$, both $\mathrm{Al}$ - and $\mathrm{OH}$-terminations present the same value for the contact angle.

Tuning of the Hydrophobicity. After assessing the factors that govern the wettability of the stoichiometric systems, we have considered the possibility of modifying the affinity of the oxides toward water, i.e. to alter its hydrophobic/hydrophilic character. In particular, we have investigated the influence of surface reduction and doping on the wetting behavior and the structure at the interface. A summary of the results is presented in Table 3 and Figure 5. Water wetting on several reducible

Table 3. Calculated versus Experimental Contact Angles, in Degrees, for the Different Materials in the Present Study ${ }^{a}$

\begin{tabular}{lllrr}
\multicolumn{1}{c}{ system } & \multicolumn{1}{c}{ doping } & \multicolumn{1}{c}{$d_{\mathrm{Me}-\mathrm{Me}}$} & \multicolumn{1}{c}{$\theta^{\text {cal }}$} & \multicolumn{1}{c}{$\theta^{\text {exp }}$} \\
$\alpha \mathrm{Al}_{2} \mathrm{O}_{3}(0001)$ & stoichiometric & 4.803 & 0.0 & $<10 \pm 4$ \\
$\mathrm{CeO}_{2}(111)$ & stoichiometric & 3.890 & 99.9 & $103 \pm 2$ \\
& $\mathrm{OH}$ & 3.890 & 0.0 & $15 \pm 6$ \\
& $\mathrm{La}$ & 3.927 & 98.9 & \\
& $\mathrm{Zr}$ & 3.772 & 112.9 & \\
$\mathrm{Nd}_{2} \mathrm{O}_{3}$ (0001) & stoichiometric & 3.860 & 103.2 & $101 \pm 3$ \\
${ }^{a} \mathrm{The} \mathrm{experiments}^{2}$ correspond to data from refs 12 and 22. \\
\hline
\end{tabular}

oxides can be modified by illumination; $;^{22,23,64}$ in fact, light can generate oxygen vacancies leading to surface reduction, thus modifying the outermost surface layer. Also, these vacancies on the surface of oxides alter the interfacial structure, as these sites are responsible for the dissociation of water and hydroxyl pinning in the form of $\mathrm{O}_{\text {lat }} \mathrm{H}^{21,65}$ Hydroxyl groups on the surface have been proposed to affect wetting for adsorption on metals. ${ }^{21}$ Recent STM experiments, combined with atomistic simulations, have indicated that, at least for small water clusters on $\mathrm{FeO}_{x}$, the effect is due to a reorientation of the water molecules; for water hexamers this can lead to nearly isoenergetic wetting and nonwetting hexameric clusters. ${ }^{30}$

The nature of the water bilayer at the interface (close to an $\mathrm{H}$-down configuration) resembles the behavior of dissociated water on $\mathrm{Ru}$, which has been claimed to be hydrophobic. ${ }^{66} \mathrm{We}$ modeled the role of vacancies at the interface as follows. We replaced one of the lattice oxygens in the $\mathrm{CeO}_{2}$ surface by an $\mathrm{O}_{\mathrm{lat}} \mathrm{H}$ group (coverage $0.25 \mathrm{ML}$ ), and examined water adsorption in the same manner as for the pristine surface.
The bilayer water interacting with the surface is pinned by the $\mathrm{O}_{\mathrm{lat}} \mathrm{H}$, orienting a flat molecule toward an acid center (see Figure 6). Hence, the perpendicular waters in the bilayer can reorganize from the typical $\mathrm{H}$-up configuration toward an $\mathrm{H}$ down one, to favor the interaction with the free basic centers on the surface. Therefore, the localized $\mathrm{O}_{\mathrm{lat}} \mathrm{H}$ centers in isolated form are better adsorption positions than the native $\mathrm{Ce}(\mathrm{IV})$ cations. These centers are good at pinning the flat water molecules and allow for the reorganization of the rest of the bilayer, maximizing the number of $\mathrm{H}$-bonds, both to the surface and within the bilayer. The bilayer can then release part of the compressive strain suffered when adsorbing on the clean $\mathrm{CeO}_{2}(110)$ surface by increasing its corrugation, thus reducing the energy penalty associated with strain. The resulting structure turns out to be wetting in nature due to the combination of all these factors. This can be further explained in terms of ref 12 description summarized in Figure 1. Indeed, the $\mathrm{Ce}(\mathrm{IV})$ centers are slightly below the plane formed by the oxygen atoms of the $\mathrm{CeO}_{2}(111)$ surface, and in this way they can be seen as screened by the anions. The sticking $\mathrm{O}_{\text {lat }} \mathrm{H}$ centers are, in comparison, less physically hindered and thus more available for interaction.

The first water bilayer is strongly bound to the surface $\left(-0.56 \mathrm{eV} / \mathrm{H}_{2} \mathrm{O}\right)$ with respect to the free-standing water bilayer. Further adsorption leaves an extra exothermic contribution of $0.15 \mathrm{eV} / \mathrm{H}_{2} \mathrm{O}$. Still, water adsorption is less exothermic than the ice cohesive energy, thus resulting in a hydrophobic structure. The origin of this hydrophobicity is 2fold. On the one hand, the lattice hydroxyl blocks the approach of the flat molecules to the surface, while the rest of free oxygens lock the incoming $\mathrm{H}$-up and $\mathrm{H}$-down water molecules. The corrugation turns out to be of $1.275 \AA$ and, the corresponding contact angle is close to zero degrees. This accounts for the perfect wetting observed for oxides in which vacancies can be formed by chemical and photochemical methods. ${ }^{16,22,23,64}$ When exposed to air conditions, a certain amount of oxygen can be dissolved in water; this oxygen can lead to the healing of the vacancies, thus reverting the termination to stoichiometric one. As a result, the pinning $\mathrm{O}_{\mathrm{lat}} \mathrm{H}$ does not exist and the wetting/dewetting is fully reversible.

An alternative way to modify the state of the surface is through aliovalent $(\mathrm{La})$ and isovalent $(\mathrm{Zr})$ cation doping, as impurities are known to alter the local geometric and electronic structure of materials. ${ }^{56}$ Hence, we doped ceria with both La (larger radius) and $\mathrm{Zr}$ (smaller radius) and optimized the doped bulk. Once the new (111) slab is relaxed, we calculated the adsorption energy and the contact angle $\theta$, as in the previous cases.

For ceria, an $8 \%$ substitution of Ce with La leads to a $5 \%$ strain of the surface. This is reflected in a larger $\mathrm{Ce}-\mathrm{Ce}$ mean distance compared to the undoped surface (3.927 and $3.890 \AA$, respectively). The contact angle is one degree lower compared to the latter and the ice arrangement is closer to that of the ice bulk. Moreover, larger dopants are known to accommodate the larger $\mathrm{Ce}^{3+}$ species formed upon reduction better. ${ }^{67}$ On the other hand, if we use the smaller metal $\mathrm{Zr}$ as a substituent, the final structure is compressed by around $1-2 \%$ and the $\mathrm{Ce}-\mathrm{Ce}$ distance becomes $3.772 \AA$, which also distorts the ice layer. The resulting contact angle of $113^{\circ}$ is larger than for the undoped case. It is worth noticing that $\mathrm{La}$ is formally charged +3 compared to the formal charge of +4 of Ce. This also induces an electronic effect, but our results show that this is negligible 

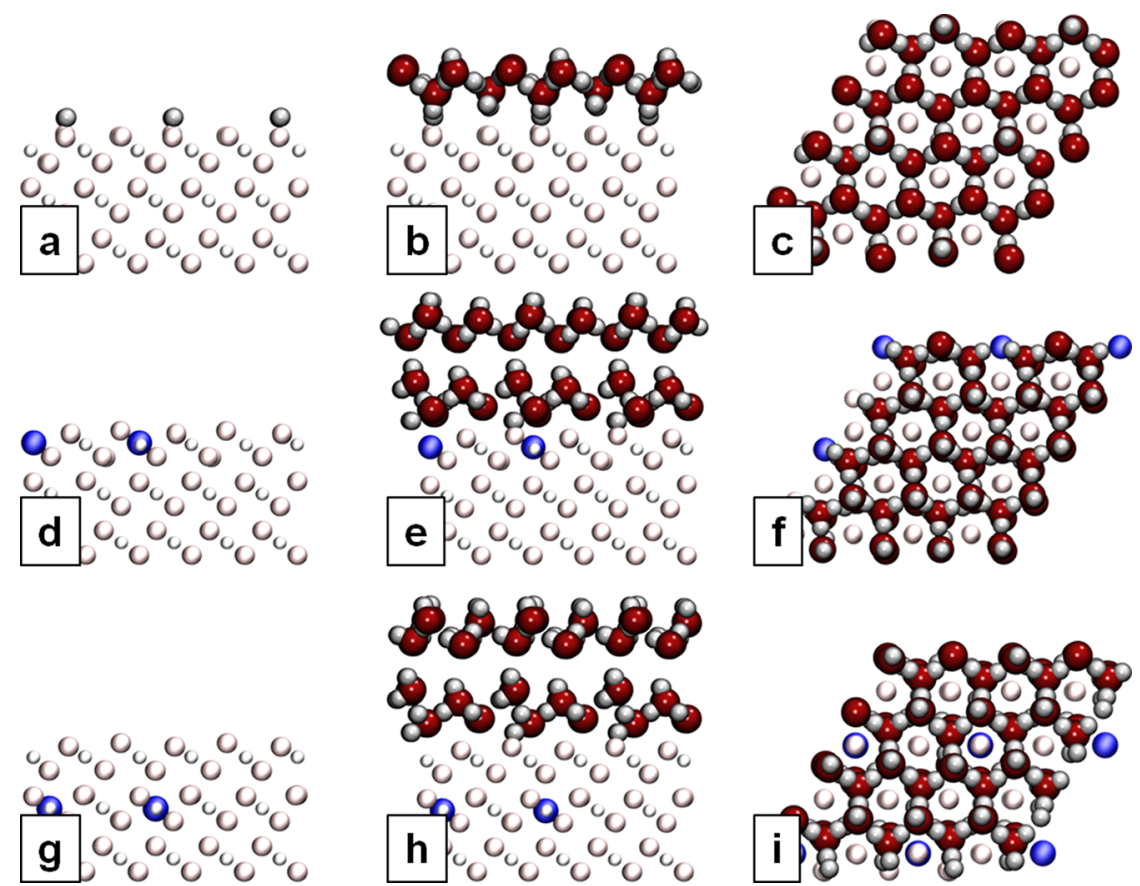

Figure 6. $\mathrm{CeO}_{2}(111)$ with $\mathrm{O}_{\mathrm{lat}} \mathrm{H}$ clean surface (a), side (b), and top (c) view in the presence of water. The $\mathrm{O}_{\mathrm{lat}} \mathrm{H}$ modifies the orientation of a flat molecule, thus strengthening the interaction with water. Clean (d), side (e), and top (f) view of La doped ceria. La, with its larger radius, determines the relaxation of the lattice and increases the mean $\mathrm{Ce}-\mathrm{Ce}$ distance, which improves the wetting of the system. When $\mathrm{Zr}$ is employed as dopant, the lattice becomes more compressed, which results in a general weakening of the interaction with the water bilayer (clean (g), side (h), and top ( $\mathrm{i})$ view of the $\mathrm{Zr}$ doped ceria).

and that the geometry of the system is the main driving force behind the wettability of the system.

Figure 5 summarizes the main contributions of geometric and electronic terms in wetting of oxide surfaces. According to our model, and provided that smaller metal-metal distances are employed on the oxides, it would result in more hydrophobic systems. This would agree with observations for $\mathrm{ZnO}^{23}$ and $\alpha-\mathrm{Fe}_{2} \mathrm{O}_{3}{ }^{68}$ for which values of the contact angle of about $160^{\circ}$ have been reported. However, for large compressions, releasing the stress through long-range reconstructions could also be possible. In any case, these surfaces, when affected by oxygen losses (vacancies) and persistent hydroxyl generation, would tend to increase their wettability.

\section{CONCLUSIONS}

Density functional theory was employed to analyze the intrinsic hydrophobicity of two REOs, that is, $\mathrm{CeO}_{2}$ and $\mathrm{Nd}_{2} \mathrm{O}_{3}$, and their wetting properties compared to those of a common, highly wettable oxide, alumina. The wetting properties can be described in terms of geometric and electronic effects. For the alumina, the almost perfect match between an ice layer and either aluminum or hydroxyl terminated surfaces leads to perfect wetting. The situation is different for the REOs. For the pristine surfaces, adsorbed ice does not match the lattice parameter of the material. As a result, the acid/base character of the surface is overruled by the geometric contribution and only part of the water molecules at the interface can dissociate. Also, the wetting behavior is univocally controlled by the relative size of the ice layer with respect to that of the surface. Therefore, the intrinsic nonwettability of the REOs can be directly assigned to a geometric control. Even doping with $\mathrm{La}$ and $\mathrm{Zr}$ does not change this nonwettability character, although a small tuning of the contact angle can be observed. In contrast, other types of surface modification can significantly alter wetting. On reducible oxides, oxygen vacancies can be easily formed. These defects can react with water and generate persistent hydroxyl groups on the surface. The chemical interaction between subsequent water layers with these hydroxyl groups changes the structure at the interface completely. The acid properties of the hydroxylated surface are more effective, both because of their localized nature and by the fact that at least some of them stick out of the surface. As a consequence, preferential adsorption points appear and, as the water bilayer can rearrange, the number and strength of the hydrogen bonds to the surface is increased. The ultimate consequence is that perfect wetting might appear due to the larger interaction at the interface. The different wetting will affect the chemistry of the materials ${ }^{11}$ therefore affecting the descriptors for these surfaces ${ }^{69}$ that might affect the new reactivity in the area of biomass. ${ }^{70}$

In summary, the present work shows that it is possible to investigate the intimate nature of complex water oxide interfaces and to determine observable parameters within the DFT approach quantitatively, like the contact angle, that are directly comparable to experiments. Simultaneously, the chemical and geometric origins of the interfacial properties can be traced back independently. The model presented paves the way for a better fundamental understanding of wettability.

\section{AUTHOR INFORMATION}

\section{Corresponding Author}

*Phone: +34 9779237. E-mail: nlopez@iciq.es.

\section{Notes}

The authors declare no competing financial interest. 


\section{ACKNOWLEDGMENTS}

This work has been supported by the ERC Starting Grant "Bio2chem-d: Biomass to chemicals: Catalysis design from firstprinciples for a sustainable chemical industry" (ERC-2010-StG258406). This study has also been carried out under the MINECO Proj. CTQ2012-33826. We acknowledge the Barcelona Supercomputing Centre (BSC-RES) for providing the computational resources.

\section{REFERENCES}

(1) Meuler, A. J.; Smith, J. D.; Varanasi, K. K.; Mabry, J. M.; McKinley, G. H.; Cohen, R. E. Relationships Between Water Wettability and Ice Adhesion. ACS Appl. Mater. Interfaces 2010, 2, $3100-3110$.

(2) Quéré, D. Wetting and Roughness. Annu. Rev. Mater. Res. 2008, $38,71-99$.

(3) Modern Approaches to Wettability, Theory and Applications; Schrader, M. E., Loeb, G. I., Eds.; Plenum Press: New York, 1992.

(4) de Gennes, P. G. Wetting and Roughness. Rev. Mod. Phys. 1985, 57, 827-863.

(5) Wang, R.; Hashimoto, K.; Fujishima, A.; Chikuni, M.; Kojima, E.; Kitamura, A.; Shimohigoshi, M.; Watanabe, T. Light-Induced Amphiphilic Surfaces. Nature 1997, 388, 431-432.

(6) Kuna, J. J.; Voïtchovsky, K.; Singh, C.; Jiang, H.; Mwenifumbo, S.; Ghorai, P. K.; Stevens, M. M.; Glotzer, S. C.; Stellacci, F. The Effect of Nanometre-Scale Structure on Interfacial Energy. Nat. Mater. 2009, 8, 837-842.

(7) Guo, Z.; Zhou, F.; Hao, J.; Liu, W. Stable Biomimetic SuperHydrophobic Engineering Materials. J. Am. Chem. Soc. 2005, 127, 15670-15671.

(8) Lafuma, A.; Quéré, D. Hydrophobic and Hydrophilic Interactions, Manufactured Materials. Nat. Mater. 2003, 2, 457-460.

(9) Deng, X.; Mammen, L.; Butt, H.-J.; Vollmer, D. Candle Soot as a Template for a Transparent Robust Superamphiphobic Coating. Science 2012, 335, 67-70.

(10) Tuteja, A.; Choi, W.; Ma, M.; Mabry, J. M.; Mazzella, S. A.; Rutledge, G. C.; McKinley, G. H.; Cohen, R. E. Designing Superoleophobic Surfaces. Science 2007, 318, 1618-1622.

(11) Stoerzinger, K. A.; Hong, W. T.; Azimi, G.; Giordano, L.; Lee, Y.-L.; Crumlin, E. J.; Biegalski, M. D.; Bluhm, H.; Varanasi, K. K.; Shao-Horn, Y. Reactivity of Perovskites with Water: Role of Hydroxylation in Wetting and Implications for Oxygen Electrocatalysis. J. Phys. Chem. C 2015, 119, 18504-18512.

(12) Azimi, G.; Dhiman, R.; Kwon, H.-M.; Paxson, A. T.; Varanasi, K. K. Hydrophobicity of Rare-Earth Oxide Ceramics. Nat. Mater. 2013, 12, 315-320.

(13) Tian, Y.; Jiang, L. Wetting: Intrinsically Robust Hydrophobicity. Nat. Mater. 2013, 12, 291-292.

(14) Oh, I.-K.; Kim, K.; Lee, Z.; Ko, K. Y.; Lee, C.-W.; Lee, S. J.; Myung, J. M.; Lansalot-Matras, C.; Noh, W.; Dussarrat, C.; Kim, H.; Lee, H.-B.-R. Hydrophobicity of Rare Earth Oxides Grown by Atomic Layer Deposition. Chem. Mater. 2015, 27, 148-156.

(15) Reitz, C.; Haetge, J.; Suchomski, C.; Brezesinski, T. Facile and General Synthesis of Thermally Stable Ordered Mesoporous RareEarth Oxide Ceramic Thin Films with Uniform Mid-Size to Large-Size Pores and Strong Crystalline Texture. Chem. Mater. 2013, 25, 46334642.

(16) Zheng, J.-Y.; Bao, S.-H.; Guo, Y.; Jin, P. Natural Hydrophobicity and Reversible Wettability Conversion of Flat Anatase $\mathrm{TiO}_{2}$ Thin Film. ACS Appl. Mater. Interfaces 2014, 6, 1351-1355.

(17) Fernández-Torre, D.; Kośmider, K.; Carrasco, J.; GandugliaPirovano, M. V.; Pérez, R. Insight into the Adsorption of Water on the Clean $\mathrm{CeO}_{2}(111)$ Surface with van der Waals and Hybrid Density Functionals. J. Phys. Chem. C 2012, 116, 13584-13593.

(18) Molinari, M.; Parker, S. C.; Sayle, D. C.; Islam, M. S. Water Adsorption and its Effect on the Stability of Low Index Stoichiometric and Reduced Surfaces of Ceria. J. Phys. Chem. C 2012, 116, 70737082 .
(19) Hayun, S.; Shvareva, T. Y.; Navrotsky, A. Nanoceria Energetics of Surfaces, Interfaces and Water Adsorption. J. Am. Ceram. Soc. 2011, 94, 3992-3999.

(20) Fronzi, M.; Piccinin, S.; Delley, B.; Traversa, E.; Stampfl, C. Water Adsorption on the Stoichiometric and Reduced $\mathrm{CeO}_{2}(111)$ Surface: a First-Principle Investigation. Phys. Chem. Chem. Phys. 2009, 11, 9188-9199.

(21) Salmeron, M.; Bluhm, H.; Tatarkhanov, M.; Ketteler, G.; Shimizu, T. K.; Mugarza, A.; Deng, X.; Herranz, T.; Yamamoto, S.; Nilsson, A. Water Growth on Metals and Oxides: Binding Dissociation and Role of Hydroxyl Groups. Faraday Discuss. 2009, 141, 221-229.

(22) Khan, S.; Azimi, G.; Yildiz, B.; Varanasi, K. K. Role of Surface Oxygen-to-Metal Ratio on the Wettability of Rare-Earth Oxides. Appl. Phys. Lett. 2015, 106, 061601.

(23) Feng, X.; Feng, L.; Jin, M.; Zhai, J.; Jiang, L.; Zhu, D. Reversible Super-hydrophobicity to Super-hydrophilicity Transition of Aligned ZnO Nanorod Films. J. Am. Chem. Soc. 2004, 126, 62-63.

(24) Carrasco, J.; Hodgson, A.; Michaelides, A. A molecular Perspective of Water at Metal Interfaces. Nat. Mater. 2012, 11, 667-674.

(25) Łodziana, Z.; Topsøe, N.-Y.; Nørskov, J. K. A Negative Surface Energy for Alumina. Nat. Mater. 2004, 3, 289-293.

(26) Sun, C.; Liu, L.-M.; Selloni, A.; Lu, G. Q. M.; Smith, S. C. Titania-Water Interactions: a Review of Theoretical Studies. J. Mater. Chem. 2010, 20, 10319-10334.

(27) Liu, L.-M.; Zhang, C.; Thornton, G.; Michaelides, A. Structure and Dynamics of Liquid Water on Rutile $\mathrm{TiO}_{2}(110)$. Phys. Rev. B: Condens. Matter Mater. Phys. 2010, 82, 161415.

(28) Wirth, J.; Saalfrank, P. The Chemistry of Water on alumina: Kinetics and Nuclear Quantum Effects from First Principles. J. Phys. Chem. C 2012, 116, 26829-26840.

(29) Tocci, G.; Michaelides, A. Solvent-Induced Proton Hopping at a Water-Oxide Interface. J. Phys. Chem. Lett. 2014, 5, 474-480.

(30) Merte, L. R.; Bechstein, R.; Peng, G.; Rieboldt, F.; Farberow, C. A.; Zeuthen, H.; Knudsen, J.; Laegsgaard, E.; Wendt, S.; Mavrikakis, M.; Besenbacher, F. Water Clustering on Nanostructured Iron Oxide Films. Nat. Commun. 2014, 5, 4193.

(31) Giovambattista, N.; Debenedetti, P. G.; Rossky, P. J. Enhanced Surface Hydrophobicity by Coupling of Surface Polarity and Topography. Proc. Natl. Acad. Sci. U. S. A. 2009, 106, 15181-15185.

(32) Giovambattista, N.; Debenedetti, P. G.; Rossky, P. J. Effect of Surface Polarity on Water Contact Angle and Interfacial Hydration Structure. J. Phys. Chem. B 2007, 111, 9581-9587.

(33) Scatena, L. F.; Brown, M. G.; Richmond, G. L. Water at Hydrophobic Surfaces: Weak Hydrogen Bonding and Strong Orientation Effects. Science 2001, 292, 908-912.

(34) Clausen, B. S.; Schiøtz, J.; Gråbæk, L.; Ovesen, C. V.; Jacobsen, K. W.; Nørskov, J. K.; Topsøe, H. Wetting non-Wetting Phenomena During Catalysis: Evidence from in Situ On-Line EXAFS Studies of Cu-Based Catalysts. Top. Catal. 1994, 1, 367-376.

(35) Lopez, N.; Nørskov, J. K.; Janssens, T. V. W.; Carlsson, A.; PuigMolina, A.; Clausen, B. S.; Grunwaldt, J.-D. The Adhesion and Shape of Nanosized $\mathrm{Au}$ Particles in a $\mathrm{AuTiO}_{2}$ Catalyst. J. Catal. 2004, 225, $86-94$.

(36) Jensen, M. C. R.; Venkataramani, K.; Helveg, S.; Clausen, B. S.; Reichling, M.; Besenbacher, F.; Lauritsen, J. V. Morphology, Dispersion, and Stability of $\mathrm{Cu}$ Nanoclusters on Clean and Hydroxylated $\alpha$-Al2O3(0001) Substrates. J. Phys. Chem. C 2008, 112, 16953-16960.

(37) García-Mota, M.; Rieger, M.; Reuter, K. Ab Initio Prediction of the Equilibrium Shape of Supported Ag Nanoparticles on $\alpha$ $\mathrm{Al}_{2} \mathrm{O}_{3}(0001)$. J. Catal. 2015, 321, 1-6.

(38) Barmparis, G. D.; Lodziana, Z.; Lopez, N.; Remediakis, I. N. Nanoparticle Shapes by Using Wulff Constructions and FirstPrinciples Calculations. Beilstein J. Nanotechnol. 2015, 6, 361-368.

(39) Kresse, G.; Furthmüller, J. Efficient Iterative Schemes for Ab Initio Total-Energy Calculations Using a Plane-Wave Basis Set. Phys. Rev. B: Condens. Matter Mater. Phys. 1996, 54, 11169-11186. 
(40) Kresse, G.; Hafner, J. Ab Initio Molecular Dynamics for Liquid Metals. Phys. Rev. B: Condens. Matter Mater. Phys. 1993, 47, 558-561. (41) Perdew, J. P.; Burke, K.; Ernzerhof, M. Generalized Gradient Approximation Made Simple. Phys. Rev. Lett. 1996, 77, 3865-3868.

(42) Kresse, G.; Joubert, D. From Ultrasoft Pseudopotentials to the Projector Augmented-Wave Method. Phys. Rev. B: Condens. Matter Mater. Phys. 1999, 59, 1758-1775.

(43) Revilla-Lopez, G.; Lopez, N. A Unified Study for Water Adsorption on Metals: Meaningful Models from Structural Motifs. Phys. Chem. Chem. Phys. 2014, 16, 18933-18940.

(44) Wang, X.-L.; Hubbard, C. R.; Alexander, K. B.; Becher, P. F.; Fernandez-Baca, J. A.; Spooner, S. Neutron Diffraction Measurements of the Residual Stresses in $\mathrm{Al}_{2} \mathrm{O}_{3}-\mathrm{ZrO}_{2}\left(\mathrm{CeO}_{2}\right)$ Ceramic Composites. J. Am. Ceram. Soc. 1994, 77, 1569-1575.

(45) Hass, K. C. K.; Schneider, W. W. F.; Curioni, A.; Andreoni, W. The Chemistry of Water on Alumina Surfaces: Reaction Dynamics from First Principles. Science 1998, 282, 265-268.

(46) Ranea, V. A.; Carmichael, I.; Schneider, W. F. DFT Characterization of Coverage Dependent Molecular Water Adsorption Modes on $\alpha$-Al2O3(0001). J. Phys. Chem. C 2009, 113, 2149-2158.

(47) Wang, X.-G.; Chaka, A.; Scheffler, M. Effect of the Environment on $\alpha-\mathrm{Al}_{2} \mathrm{O}_{3}(0001)$ Surface Structures. Phys. Rev. Lett. 2000, 84, 36503653.

(48) Łodziana, Z.; Nørskov, J. K.; Stoltze, P. The Stability of the Hydroxylated (0001) Surface of $\alpha-\mathrm{Al}_{2} \mathrm{O}_{3}$. J. Chem. Phys. 2003, 118, 11179-11188.

(49) García-Melchor, M.; López, N. Homolytic Products from Heterolytic Paths in H2 Dissociation on Metal Oxides: The Example of CeO2. J. Phys. Chem. C 2014, 118, 10921-10926.

(50) Kümmerle, E.; Heger, G. The Structures of $\mathrm{Ce} 2 \mathrm{O} 3+\delta, \mathrm{Ce}_{7} 7_{12}$, and $\mathrm{Ce}_{11} \mathrm{O}_{20}$. J. Solid State Chem. 1999, 147, 485-500.

(51) Cococcioni, M.; de Gironcoli, S. Linear response Approach to the Calculation of the Effective Interaction Parameters in the LDA+U Method. Phys. Rev. B: Condens. Matter Mater. Phys. 2005, 71, 035105.

(52) Ganduglia-Pirovano, M. V.; Da Silva, J. L. F.; Sauer, J. DensityFunctional Calculations of the Structure of Near-Surface Oxygen Vacancies and Electron Localization on $\mathrm{CeO}_{2}(111)$. Phys. Rev. Lett. 2009, 102, 026101.

(53) Brunauer, S.; Emmett, P. H.; Teller, E. Adsorption of Gases in Multimolecular Layers. J. Am. Chem. Soc. 1938, 60, 309-319.

(54) Amrute, A. P.; Mondelli, C.; Moser, M.; Novell-Leruth, G.; López, N.; Rosenthal, D.; Farra, R.; Schuster, M. E.; Teschner, D.; Schmidt, T.; Pérez-Ramírez, J. Performance, Structure, and Mechanism of $\mathrm{CeO}_{2}$ in $\mathrm{HCl}$ Oxidation to $\mathrm{Cl}_{2}$. J. Catal. 2012, 286, 287-297.

(55) Lopez, N.; Prades, J. D.; Hernandez-Ramirez, F.; Morante, J. R.; Pan, J.; Mathur, S. Bidimensional Versus Tridimensional Oxygen Vacancy Diffusion in SnO2-x Under Different Gas Environments. Phys. Chem. Chem. Phys. 2010, 12, 2401-2406.

(56) Farra, R.; García-Melchor, M.; Eichelbaum, M.; Hashagen, M.; Frandsen, W.; Allan, J.; Girgsdies, F.; Szentmiklósi, L.; López, N.; Teschner, D. Promoted Ceria: A Structural, Catalytic, and Computational Study. ACS Catal. 2013, 3, 2256-2268.

(57) Adachi, G.-Y.; Imanaka, N. The Binary Rare Earth Oxides. Chem. Rev. 1998, 98, 1479-1514.

(58) Young, T. An Essay on the Cohesion of Fluids. Philos. Trans. $R$. Soc. London 1805, 95, 65-87.

(59) Della Volpe, C.; Maniglio, D.; Siboni, S.; Morra, M. An Experimental Procedure to Obtain the Equilibrium Contact Angle from the Wilhelmy Method. Oil Gas Sci. Technol. 2001, 56, 9-22.

(60) Van Oss, C.; Giese, R.; Wentzek, R.; Norris, J.; Chuvilin, E. Surface Tension Parameters of Ice Obtained from Contact Angle Data and from Positive and Negative Particle Adhesion to Advancing Freezing Fronts. J. Adhes. Sci. Technol. 1992, 6, 503-516.

(61) Lange, B.; Posner, R.; Pohl, K.; Thierfelder, C.; Grundmeier, G.; Blankenburg, S.; Schmidt, W. G. Water Adsorption on Hydrogenated Si(111) Surfaces. Surf. Sci. 2009, 603, 60-64.

(62) Giordano, L.; Goniakowski, J.; Suzanne, J. Partial Dissociation of Water Molecules in the Water Monolayer Deposited on the $\mathrm{MgO}$ (100) Surface. Phys. Rev. Lett. 1998, 81, 1271-1273.
(63) Capdevila-Cortada, M.; García-Melchor, M.; López, N. Unraveling the Structure Sensitivity in Methanol Conversion on $\mathrm{CeO}_{2}$ : A DFT+U Study. J. Catal. 2015, 327, 58-64.

(64) Feng, X.; Zhai, J.; Jiang, L. The Fabrication and Switchable Superhydrophobicity of $\mathrm{TiO}_{2}$ Nanorod Films. Angew. Chem., Int. Ed. 2005, 44, 5115-5118.

(65) Schaub, R.; Thostrup, P.; López, N.; Lægsgaard, E.; Stensgaard, I.; Nørskov, J. K.; Besenbacher, F. Oxygen Vacancies as Active Sites for Water Dissociation on Rutile $\mathrm{TiO}_{2}(110)$. Phys. Rev. Lett. 2001, 87, 266104.

(66) Haq, S.; Hodgson, A. Multilayer Growth and Wetting of $\mathrm{Ru}(0001)$. J. Phys. Chem. C 2007, 111, 5946-5953.

(67) Balducci, G.; Islam, M. S.; Kašpar, J.; Fornasiero, P.; Graziani, M. Reduction Process in $\mathrm{CeO}_{2} \mathrm{MO}$ and $\mathrm{CeO}_{2} \mathrm{M}_{2} \mathrm{O}_{3}$ Mixed Oxides: A Computer Simulation Study. Chem. Mater. 2003, 15, 3781-3785.

(68) Yan, B.; Tao, J.; Pang, C.; Zheng, Z.; Shen, Z.; Huan, C. H. A.; $\mathrm{Yu}, \mathrm{T}$. Reversible UV-Light-Induced Ultrahydrophobic-to-Ultrahydrophilic Transition in an $\mathrm{Fe}_{2} \mathrm{O}_{3}$ Nanoflakes Film. Langmuir 2008, 24, 10569-10571.

(69) Capdevila-Cortada, M.; Lopez, N. Descriptor Analysis in Methanol Conversion on Doped $\mathrm{CeO}_{2}$ (111): Guidelines for Selectivity Tuning. ACS Catal. 2015, 5, 6473-6480.

(70) Haider, M. H.; Dummer, N. F.; Knight, D. W.; Jenkins, R. L.; Howard, M.; Moulijn, J.; Taylor, S. H.; Hutchings, G. J. Efficient green methanol synthesis from glycerol. Nature Chem. 2015, 7, 1028-1032. 